\title{
Professionalism in the intimate examination: How healthcare practitioners feel about having chaperones present during an intimate consultation and examination
}

\author{
Y Guidozzi, J Gardner, A Dhai \\ Steve Biko Centre for Bioethics, Faculty of Health Sciences, University of the Witwatersrand, Johannesburg \\ Y Guidozzi, PhD candidate, BSc Nurs, LLB, MBA \\ J Gardner, PhD candidate, MSc Med (Bioethics \& Health Law), BA \\ A Dhai, PhD candidate, MB ChB, FCOG (SA), LLM, PG Dip Int Res Ethics
}

Corresponding author: Y Guidozzi (Yolande.Guidozzi@wits.ac.za)

\begin{abstract}
Background. Despite the clear prohibition against sexual relations with one's patients, complaints of a sexual nature against practitioners registered with the Health Professions Council of South Africa (HPCSA) have been increasing. The HPCSA does not provide ethical guidelines regarding the use of a chaperone during intimate examinations.

Aims. (i) To ascertain how a group of medical practitioners felt about the presence of chaperones during the consultation and intimate examination of patients; (ii) to determine whether they currently engage the services of chaperones; (iii) to assess how they felt about consensual sexual relationships between medical practitioners and their patients.

Methods. A self-administered, questionnaire-based survey was distributed to gynaecologists and medical practitioners.

Results. There was a $43 \%$ response rate with $72 \%$ of practitioners in favour of using a chaperone during an intimate examination, although only $27 \%$ always do so. Most practitioners felt that consensual sexual relationships with patients are unacceptable; $83 \%$ felt that ethical guidelines on this topic were needed.

Conclusion. The HPCSA should develop guidelines on the use of chaperones to assist practitioners. With medical litigation increasing, using chaperones will benefit patients and practitioners.

S Afr Med J 2013;103(1):25-27. DOI:10.7196/SAMJ.6224
\end{abstract}

Professionalism in the relationship between the medical practitioner and patient, and the role-based trust implicit in healthcare, do not allow the crossing of sexual boundaries. The prohibition of sexual impropriety in the practitioner-patient relationship dates back to the Hippocratic Oath, underscoring the vulnerability of patients in this unequal relationship.

Trust is a cornerstone of the practitioner-patient relationship. Where a practitioner inappropriately uses words or actions of a sexual nature with a patient, a professional boundary has been crossed and trust and patient welfare are compromised. Such violations are not necessarily confined to physical actions that are exploitative or harmful to patients. Sexual misconduct may be categorised as:

- sexual impropriety - this includes behaviour, gestures or expressions that are sexually suggestive, seductive, disrespectful of a patient's privacy or sexually demeaning to a patient

- sexual violation - this includes physical sexual contact between a practitioner and a patient, whether or not it was consensual and/or initiated by the patient. This includes any kind of genital contact or masturbation, and touching of any sexualised part of the body for purposes other than appropriate medically related examination or treatment. Exchange of prescriptions or other professional services for sexual favours is also a violation. ${ }^{1}$

Despite this codified prohibition, complaints of sexual misconduct against practitioners registered with the Health Professions Council of South Africa (HPCSA) continue to increase alarmingly. ${ }^{2}$ It is therefore prudent to consider using chaperones during intimate examinations. ${ }^{3}$ While chaperones primarily protect the patient, they also act as a risk management strategy for practitioners. ${ }^{4,5}$

The UK's General Medical Council and Royal College of Obstetricians and Gynaecologists, as well as the American Medical
Association, have long advised using a chaperone for intimate examinations. While studies show that practitioners generally see no need for this, patients, especially women, prefer to have a chaperone present where the examiner is male. ${ }^{6}$

We report on a study to determine whether a group of South African medical practitioners, mostly gynaecologists, support the idea of having a chaperone during consultations and intimate examinations. This was designed to: $(i)$ ascertain how a group of medical practitioners felt about the presence of chaperones during the consultation and intimate examination of patients, (ii) determine whether they currently engage the services of chaperones during consultations, and (iii) assess how they felt about consensual sexual relationships between medical practitioners and their patients.

\section{Methods}

This study used a self-administered, questionnaire-based survey that was pre-tested as a pilot study with several gynaecologists at the University of the Witwatersrand. Ethical clearance was granted by the University of the Witwatersrand Research Ethics Committee (Medical). Prospective participants were recruited at 3 meetings attended by gynaecologists and general medical practitioners who conducted gynaecological examinations. A total of 503 practitioners were present at these gatherings, which were held in Durban (an update meeting and a national conference) and Cape Town (an update meeting).

A total of 218 (43\%) questionnaires were completed, of which 179 came from gynaecologists. As there are about 550 practising gynaecologists in South Africa, this survey therefore obtained opinions from $33 \%$ of them. 
After primary analysis, the analysis was demographically stratified by site, age, gender, field of practice, practice type (public/private) and place of practice (urban/rural/semi-urban). Tests for significant relationships were carried out using Pearson's $\chi^{2}$ test at the $95 \%$ confidence level. The strength of the associations was determined by Cramér's V.

\section{Results and discussion}

Of the respondents, $15 \%$ were general practitioners, $82 \%$ were gynaecologists and 3\% did not indicate their field of practice; $84 \%$ were between 30 and 60 years old and 36\% were female; $59 \%$ were from the private sector and $37 \%$ from the public sector; $2 \%$ worked in both sectors and 3\% did not indicate their type of practice; $81 \%$ worked in urban areas, $11 \%$ in semi-urban, and $7 \%$ in rural areas.

\section{Attitude to presence of chaperones during consultation} and intimate examination

Many respondents felt it necessary to have a chaperone present during a consultation and intimate examination of patients: $33 \%$ felt it is always necessary, $39 \%$ felt it is sometimes necessary and $28 \%$ felt it is not necessary.

There was a significant, but weak, association (Pearson's $\chi^{2}$ test, $p=0.024$; Cramér's $V=0.18$ ) between respondents' gender and whether they felt it necessary to have a chaperone present: males were more likely to agree than not, while the reverse was true for females (Fig. 1). The Medical Protection Society cautions that gender should not lead to a false sense of security as complaints are received against practitioners of the same gender as the patient. ${ }^{4}$

Of the female respondents, $42 \%$ felt it necessary to have a chaperone present sometimes, and suggested that the need for a chaperone be decided on a case-by-case basis. The $21 \%$ who indicated that chaperones are always necessary stated that this was for medico-legal reasons, or to assist with interpretation and translation, particularly in rural areas. Of males, $40 \%$ felt that it was necessary to have a chaperone present for medico-legal reasons or because it makes patients feel less anxious and more comfortable. While $23 \%$ of males felt it unnecessary to have a chaperone present, they gave no clear reasons to support their opinions. Females, however, said that they did not consider the situation to be threatening to themselves or the patients. A similar percentage of males (37\%) and females $(42 \%)$ feel that a chaperone is sometimes necessary, reinforcing the suggestion that using a chaperone should be decided on a case-by-case basis.

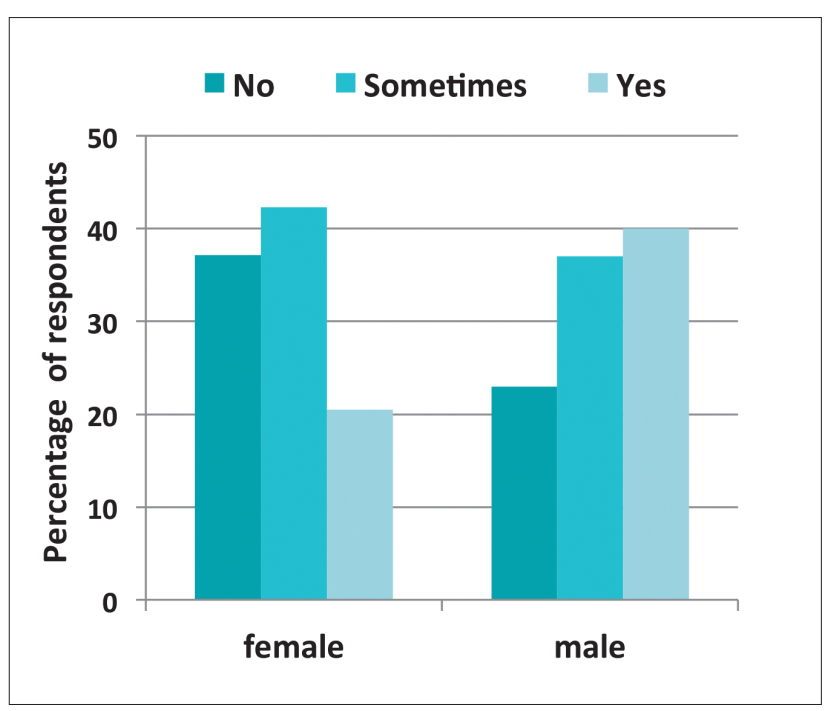

Fig. 1. Responses by gender to the question: 'Do you feel it is necessary to have a chaperone present during the consultation?'
Current use of chaperones during consultations

Of the respondents, $38 \%$ indicated that they sometimes offer patients chaperones during consultations; $35 \%$ did not offer chaperones; $27 \%$ always offer chaperones; and $2 \%$ did not answer the question.

There was a significant moderate association between the degree to which respondents felt it necessary to offer a chaperone and whether one is offered (Pearson's $\chi^{2}$ test, $p<0.001$, Cramér's V $=0.45$ ). There was a significant, but weak, association (Pearson's $\chi^{2}$ test, $p=0.021$; Cramér's V $=0.19$ ) between whether patients were offered chaperones and the gender of the respondents: the proportion by which more females responded 'no' than males was greater than expected. Female respondents also felt that because of their gender, their patients would not feel threatened by them.

Multinomial logistic regression analysis showed that males were 3.0 times more likely than females to always offer chaperones to patients, and 2.7 times more likely to offer them 'sometimes'. Respondents in the public sector were 3.3 times more likely to offer chaperones than those in the private sector. Public sector practitioners may find someone to act as a chaperone (e.g. a nurse) more easily. Concerning the type of chaperones offered, $65 \%$ of respondents used only medical staff (e.g. nurses), $21 \%$ used only family or partners, and the rest used a combination of both. Compared with female practitioners, males tended to prefer using medical staff over family members.

Attitude toward consensual sexual relationships between medical practitioners and their patients

The general sentiment was that consensual sexual relationships between practitioners and patients are unacceptable, and most respondents described this as unethical behaviour on the part of the practitioner. Many respondents, both male and female, argued for the wrongness of such behaviour by refering to the purpose and nature of the practitioner-patient relationship. Examples of responses include: 'Because patients are in a weak position.; 'Patients come to a practitioner for help, not for sex.'; 'A relationship brings emotional issues into the consultation and examination which clouds clinical judgment.'; and 'The profession needs to be held in very high esteem and practitioners must be mature enough to overcome unpleasant behaviours.' Some felt that the practitioner-patient relationship should be terminated if a sexual relationship occurs, or that practitioners who engage in sexual relations with patients should be punished, e.g. by having their names removed from the register.

However, a few practitioners did not regard a consensual sexual relationship between practitioners and patients as ethically problematic, especially if the practitioner-patient relationship is subsequently terminated. Comments from female respondents included: 'It is a fundamental right to have a consensual sexual relationship.; 'It depends under what circumstances the relationship was developed.'; and 'As long as the practitioner is not unprofessional and the relationship develops like any other relationship it is not a problem.' Comments from male respondents included: 'It is their own business and their reputations will catch up with them.; 'The sexual relationship should only take place outside the practice.; and 'We should not interfere with two consenting adults.'

Need for professional ethical guidelines on the presence of a chaperone and consensual sexual relationships

Having a chaperone present during a consultation with patients was thought to be professional practice by $60 \%$ of respondents, consistent with the number who felt a chaperone was necessary (significant strong association: Pearson's $\chi^{2}$ test, $p<0.001$; Cramér's $V=0.62$ ) and those who offered a chaperone (significant moderate association: Pearson's $\chi^{2}$ test, $p<0.001$; Cramér's V $=0.34$ ). 
There was a significant moderate association (Pearson's $\chi^{2}$ test, $p<0.001$; Cramér's $\mathrm{V}=0.30$ ) between respondents' age and whether they felt that having a chaperone present is professional: younger respondents were more in agreement than older respondents. This could be because younger practitioners have been educated in a patient-centred climate, rather than the traditional paternalistic approach to medical practice, which has also brought with it the idea of 'defensive medicine'.

Of the respondents, $83 \%$ considered it necessary to have ethical guidelines on the presence of chaperones during an intimate examination, while $80 \%$ felt it was necessary to have guidelines on consensual sexual relations between medical practitioners and their patients. However, 20\% thought that there was no need to draft such guidelines.

Regarding the necessity of guidelines on the presence of chaperones during intimate examinations, there was no significant association between respondents' opinions and their field of practice, practice type or place of practice. However, there was a significant, but weak, association with age, with more respondents $>50$ years answering that guidelines are not needed (Pearson's $\chi^{2}$ test, $p=0.007$; Cramér's $V=0.22$ ).

More respondents in the public than in the private sector felt that ethical guidelines are needed; although in both sectors the proportion of respondents who felt that guidelines are needed was very high. Public sector practitioners were 3.3 times more likely to indicate that having ethical guidelines was necessary than private sector respondents.

There was a significant but weak association (Fisher's exact test, $p=0.034$; phi coefficient $=0.15$ ) between respondents' gender and whether they felt ethical guidelines are necessary. Despite women's attitudes to the use and presence of chaperones, more women than men thought guidelines were necessary.

There were also associations between whether respondents felt it necessary to have ethical guidelines and their attitudes to offering a a chaperone. Those who felt the guidelines unnecessary tended:

- not to offer a chaperone, and vice versa (a significant but weak association)

- not to feel it necessary to offer a chaperone and vice versa (a significant but moderate association)

- not to feel it professional practice to have a chaperone and vice versa (a significant but moderate association).

Several respondents stated that since there is a rule that consensual sexual relationships are not allowed, there is no need for guidelines. An HPCSA general rule in booklet 1, paragraph 5.2.4, states, 'Healthcare practitioners should ... avoid improper relationships with their patients, their patients' friends or their patients' family members (for example, sexual relationships or exploitative financial arrangements).? Also, in terms of the Hippocratic Oath, practitioners are to keep themselves far from all intentional ill-doing and all seduction and especially from the pleasure of love with women or men.8

\section{Conclusion}

The HPCSA and international ethics codes and guidelines clearly prohibit sexual relationships between medical practitioners and patients, but this has not prevented complaints of sexual misconduct against medical practitioners in South Africa. This research established that medical practitioners, or at least gynaecologists, support the development and production of ethical guidelines on the use of chaperones.

However, these guidelines must be reasonable and take into account the challenges of making it a rule to always use a chaperone, including costs and ethical issues. The presence of a third person, even a family member, may undermine the relationship of trust between practitioner and patient, especially where that presence is enforced on the relationship. Patients should also remain free to decide whether they wish to have a chaperone or not. Therefore, although guidelines are generally considered desirable, they cannot be crafted in isolation from the issues.

In general, practitioners felt that the benefits of ethical guidelines on the use of chaperones outweigh the disadvantages. With medical litigation increasing, having chaperones has clear benefits both for individual patients and practitioners and society at large. A chaperone could be necessary in certain situations to protect the practitionerpatient relationship. While some practitioners are guilty of boundary crossing and relationship violations, patients also sometimes falsely accuse their practitioners of sexual impropriety, including rape. Hence the presence of a chaperone would give practitioners protection that is necessary in current practice. ${ }^{7}$ According to the Medical Protection Society, practitioners rarely face allegations of sexual impropriety if a chaperone is present. A chaperone's presence also acknowledges the patient's vulnerability, and provides emotional comfort and reassurance. The chaperone could also help the patient undress, assist the practitioner during the examination, and act as an interpreter. ${ }^{9}$

While the practitioners in our study supported the formulation of guidelines for consensual sexual relationships between health professionals and patients, the HPCSA general rules already indicate that this is unethical behaviour. Clearly this rule is not widely known and deserves more than a mention in the 'general rules' section. The HPCSA also does not address the use of chaperones.

The question is, should a separate booklet be published to deal with intimate examination and consensual sexual relationships specifically, or should the information be incorporated as a subsection in a guideline booklet? We suggest the latter, inserting the new guidelines in the HPCSA Guidelines for Reproductive Health, ${ }^{10}$ which the HPCSA should then market widely. The range of HPCSA booklets could also be cross-referenced so that practioners can easily identify and implement these guidelines. This inclusion in the HPCSA guidelines would put South Africa's ethical standards on par with international ethical standards, such as those of the UK and USA, where the use of chaperones has been recommended or is required.

Acknowledgments. We thank Professor S Moodley, Professor P Moodley, Professor J Bagratee, Professor Z van der Spuy, Dr P Gaylard, Professor M Vorster and Dr G Howarth for their kind assistance during the research.

\section{References}

1. Addressing Sexual Boundaries: Guidelines for State Medical Boards. Washington DC: Federation of State Medical Boards of the United States, 2006. http://www.fsmb.org.pdf/GRPOL_Sexual\%20 Boundaries (accessed 28 March 2011)

2. Dhai, A. Why do I need a chaperone? HPCSA: The Bulletin 2011:28-29.

3. Dhai A, Gardner I, Guidozzi Y, Howarth G, Vorster M. Professionalism and the intimate examination - are chaperones the answer? S Afr Med J 2011;101(11):814-816.

4. Pydah KL, Howard J. The awareness and use of chaperones by patients in an English general practice. J Med Ethics 2010;36(8):512-513.

5. Suk Yook H, Yung Jang K, Lee H. Chaperone: For or against doctors. Yonsei Med J 2009;50(4):599-600 http://dx.crossref.org/10.3349\%2Fymj.2009.50.4.599

6. Newton DC, Chen MY, Cummings R, Fairley CK. Recommendations for chaperoning in sexual health settings. Sex Health 2007;4(3):207.

7. HPCSA Booklet 1: General Ethical Guidelines for the Health Care Professions. Pretoria: HPCSA 2008:6.

8. The Hippocratic Oath. In: Biomedical Ethics, 5th ed. Mappes TA, Degrazia D, eds. Boston: McGrawHill, 2001:66.

9. Medical Protection Society Fact Sheet. http://www.mps.org uk (accessed February 2010)

10. HPCSA Booklet 13: Guidelines on Reproductive Health Management. Pretoria: HPCSA, 2008. 